Supporting Information

\title{
Nonlinearly Frequency-Adaptive, Self-Powered, Proton-Driven Somatosensor Inspired by Human Mechanoreceptor
}

Kyoung-Yong Chun, Young Jun Son, Seunghwan Seo, Ho Jung Lee, and Chang-Soo Han*

School of Mechanical Engineering, College of Engineering, Korea University, Anam-Dong,

Seongbuk-Gu, Seoul 136-713, Republic of Korea 

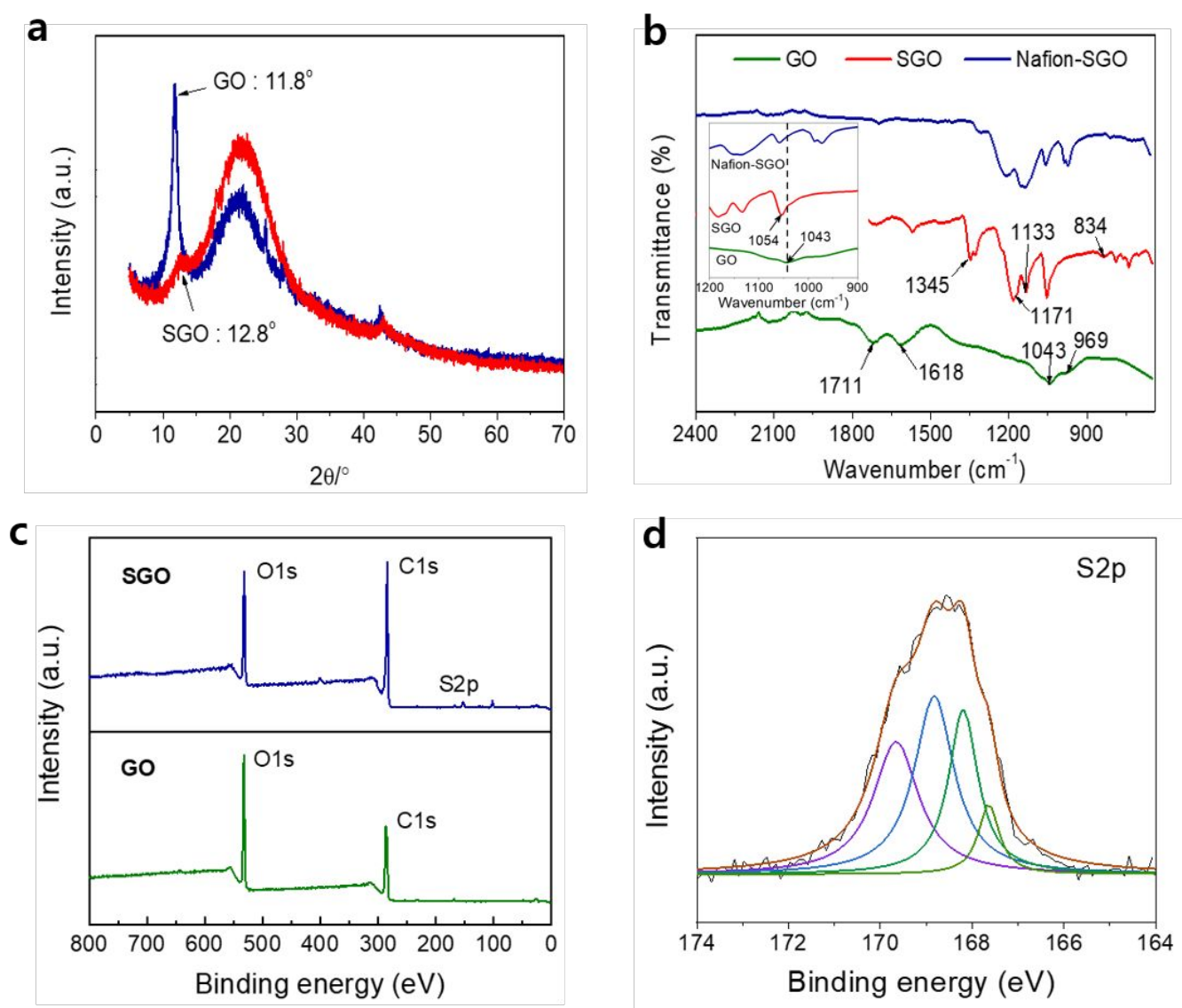

Figure S1. Analysis of SGO. (a) X-ray diffraction (XRD), (b) Fourier-transform infrared spectroscopy (FT-IR) and (c), (d) x-ray photoelectron spectroscopy (XPS). XRD analysis was performed to study the structural change of the graphene oxide (GO) and sulfonated-graphene oxide (SGO). The GO exhibits a diffraction peak at $2 \theta=11.8^{\circ}$ with an average interlayer distance of $0.77 \mathrm{~nm}$ calculated by Bragg's law $(\lambda=2 \mathrm{~d} \sin \theta)$. After sulfonation, the SGO exhibits a diffraction peak at $2 \theta=12.8^{\circ}$, which indicates that the interlayer spacing decreases to $0.69 \mathrm{~nm}$. The structures of the graphene oxide derivatives were characterized by the FT-IR spectra. In the XPS spectra of the GO and SGO, a peak at S 2p located at $168 \mathrm{eV}$ confirming the presence of the $-\mathrm{SO}_{3} \mathrm{H}$ group for the SGO. Deconvoluted peaks of S $2 \mathrm{p}$ spectra of the SGO are in the range of $167 \mathrm{eV}-170 \mathrm{eV}$. 

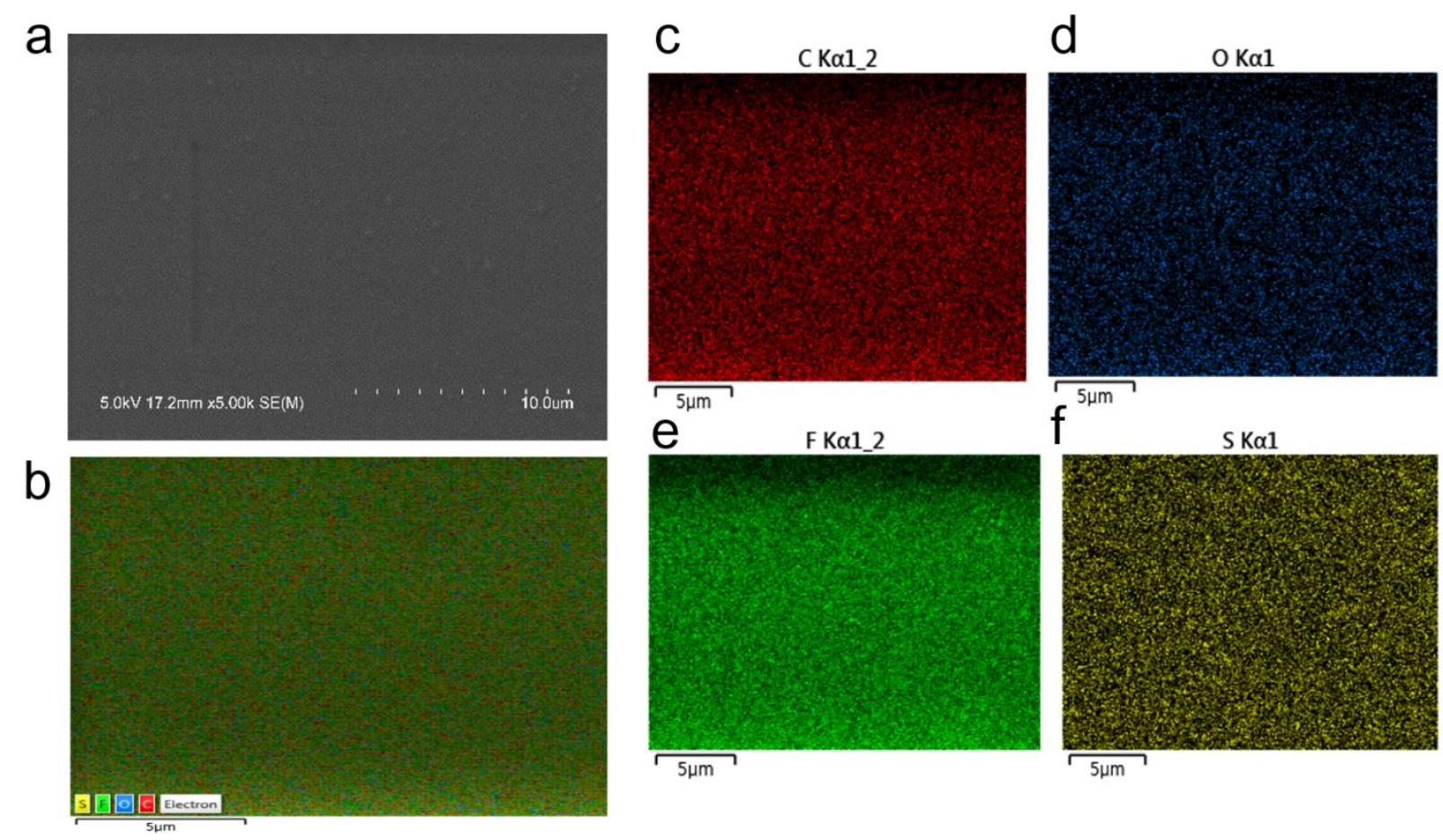

Figure S2. The images obtained using scanning electron microscopy (SEM): (a) SEM images of the surface of the Nafion/SGO 12 composite film. (b) Elements mapping of the same surface of the Nafion/SGO 12 composite film. (c), (d), (e), and (f) Mapping of carbon, oxygen, fluorine, and sulfur (approximately 2 wt.\%), respectively. Sulfonation is well confirmed with fine distribution of sulfur in surface of NF-SGO 12. 

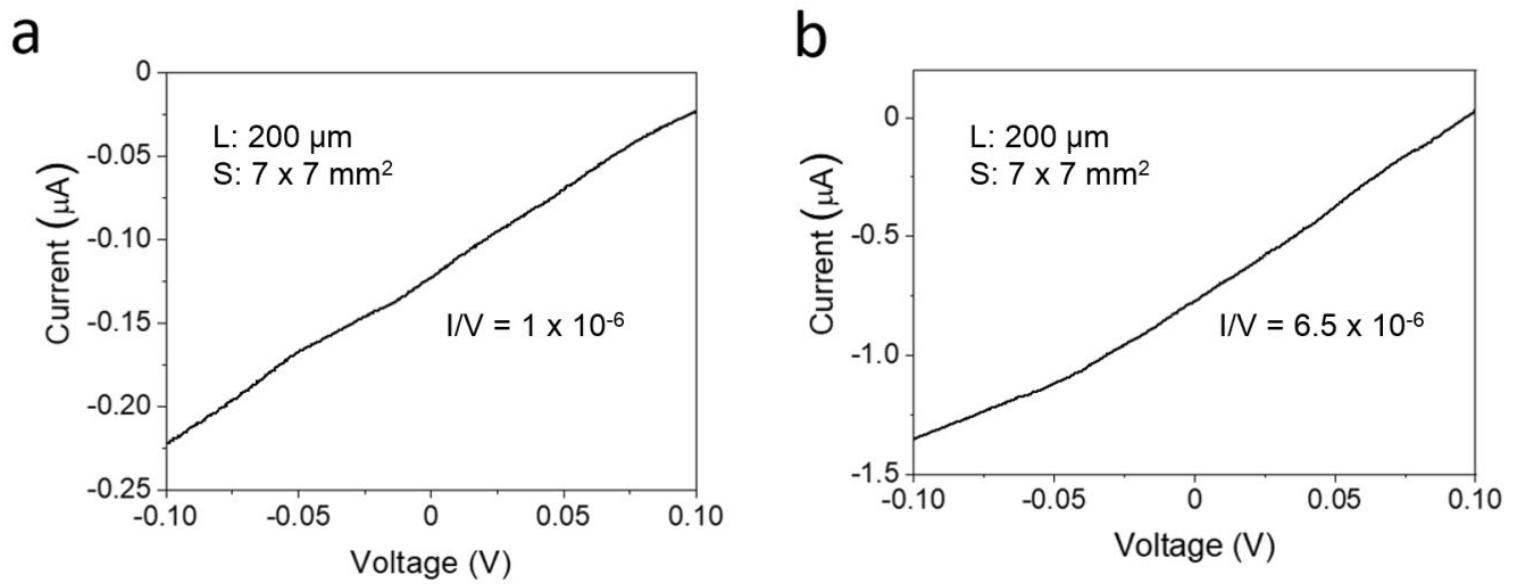

Figure S3. I-V plot of the NF-SGO. The electron conductivity calculated from the average I-V curve obtained from the cyclic voltammetry in potentiostat was approximately $4 \times 10^{-8} \mathrm{~S} / \mathrm{cm}$ for a dry condition (a) and approximately $2.6 \times 10^{-7} \mathrm{~S} / \mathrm{cm}$ for a wet condition (b) at room temperature. Electrical conductivity is calculated by the equation $\delta=\mathrm{L} /(\mathrm{R} \mathrm{S})$, where $\mathrm{L}$ is the thickness of the NF-SGO, R is the resistance by determined from slope of IV curves, and $\mathrm{S}$ is the surface area of the interface between the NF-SGO and the electrodes. 


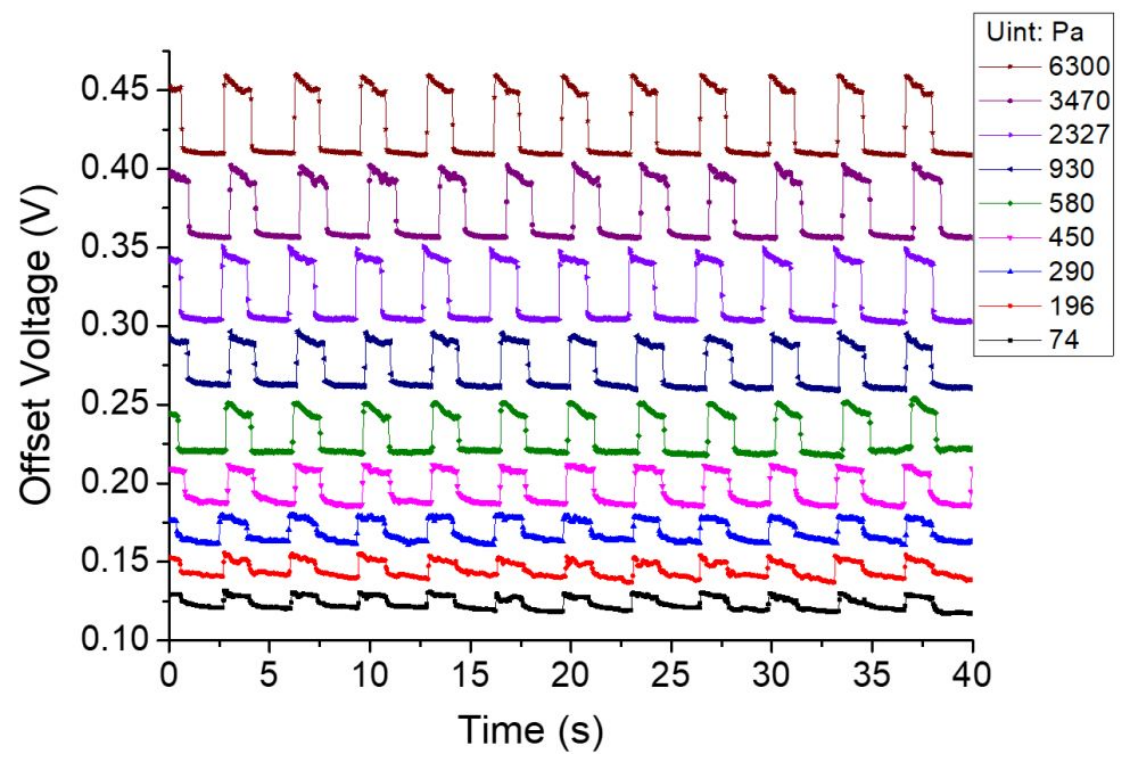

Figure S4. Continuous voltage signals indicating that the output voltage increased with increasing pressure, which shows static type depending on pressure. The value of the voltage change with respect to pressure was obtained using a device such as a conventional home-made force sensor. 

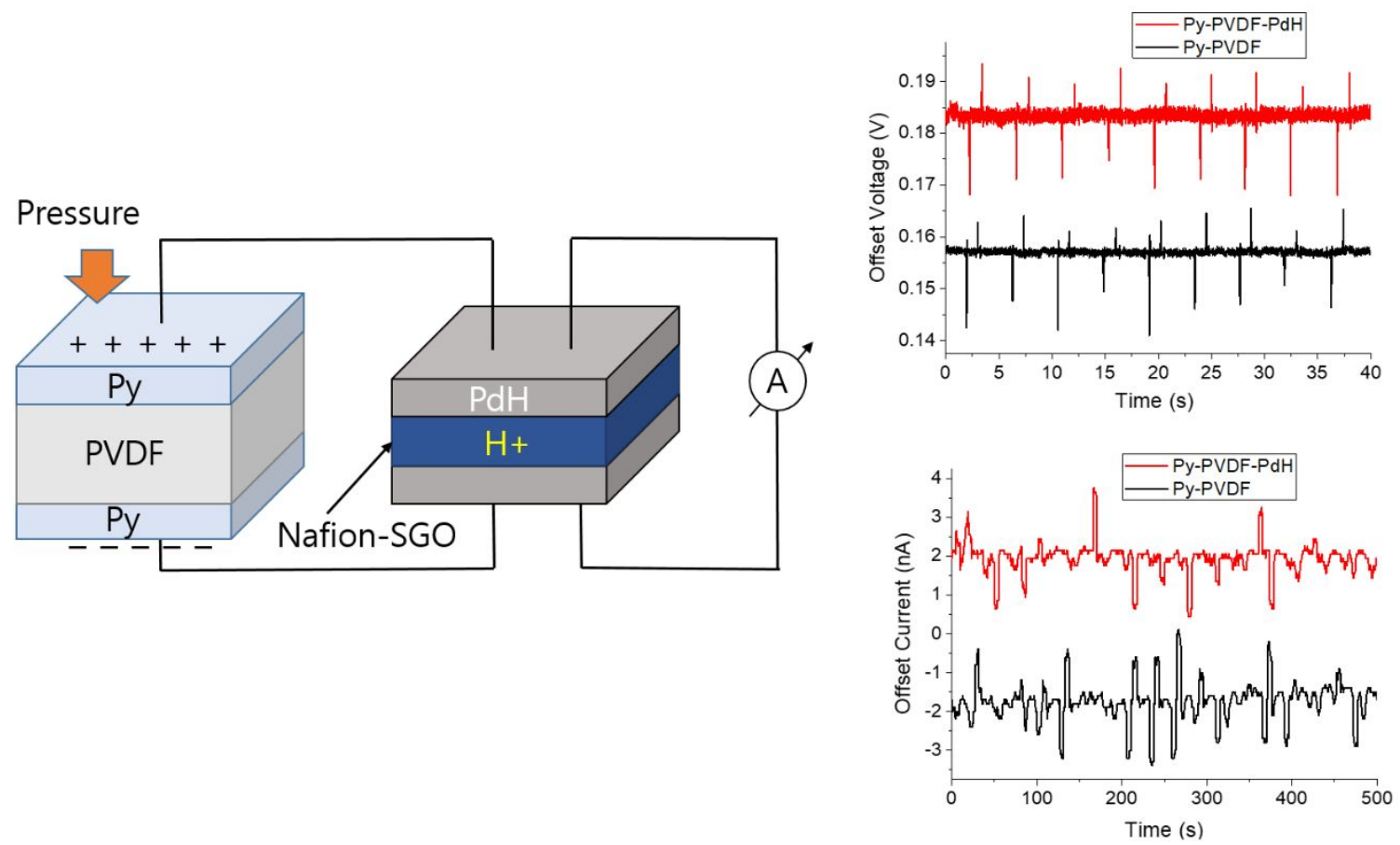

Figure S5. Experiments on pp-PVDF and $\mathrm{PdH}_{\mathrm{x}}$ in a parallel circuit compared to that on pp-PVDF itself during pressure application. It indicates that the operation of voltage and current generated by pp-PVDF under pressure is fully performed through proton injecting electrode and proton transfer medium. 


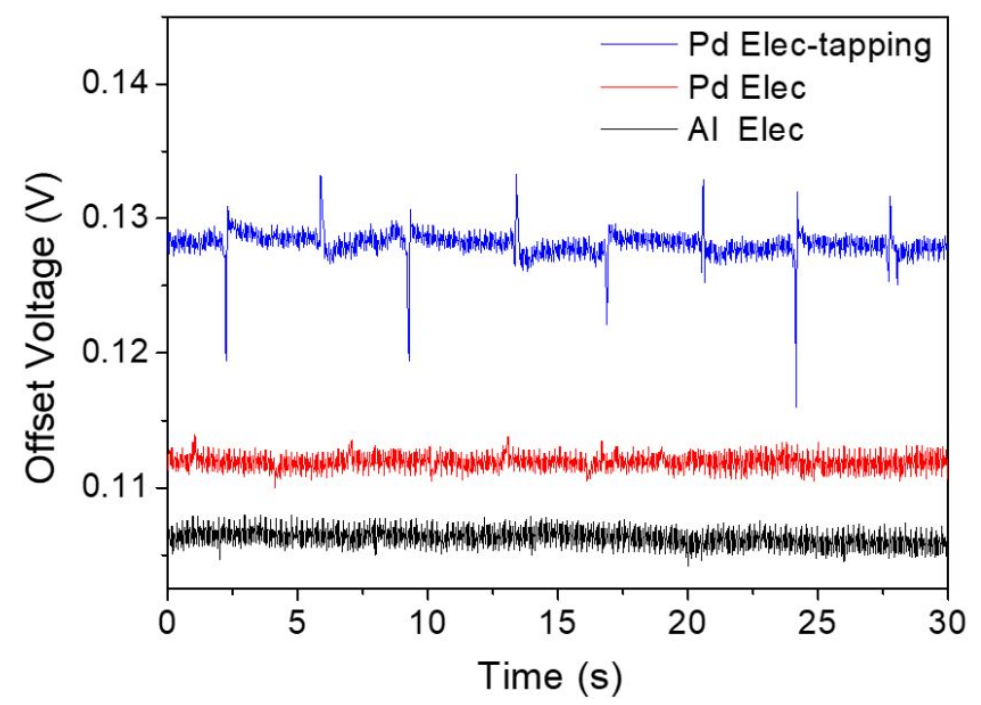

Figure S6. Electrical response for other electrodes ( $\mathrm{Pd}, \mathrm{Al})$. The pressure sensing is difficult to observe when Pd and $\mathrm{Al}$ electrodes are used, even at $500 \mathrm{~Pa}$. 